\title{
Reliability and validity of tympanic temperature measurement in persons with high spinal cord injuries
}

\author{
A Chu ${ }^{1}$ and RS Burnham ${ }^{2}$ \\ ${ }^{1}$ Resident in Physical Medicine and Rehabilitation, ${ }^{2}$ Assistant Professor of Medicine, University of Alberta, \\ Canada
}

\begin{abstract}
Tympanic temperature measurements in the able bodied have been well studied and validated in previous investigations. This validation has not been studied in individuals with high spinal cord injuries where autonomic control is different above and below the level of the lesion, which makes the accuracy of this measurement for core body temperature questionable. In this correlational study we look at the reliability and validity of tympanic temperature measurement in individuals with high spinal cord injuries in comparison to oral and rectal temperature measures. The rectal measurement proved to be the most reliable with an $r$ value of 0.975 overall. The oral was minimally better than the tympanic with $r$ values of 0.88 and 0.86 , respectively. The validity of the oral measure in predicting changes in the rectal temperature (gold standard) was slightly better than the tympanic with $r$ values of 0.88 and 0.77 , respectively. Both the oral and tympanic measures accurately reflected changes in the rectal (core) temperature.
\end{abstract}

Keywords: tympanic temperature; tetraplegia; spinal cord injury; rectal; oral

\section{Introduction}

The measurement of body temperature is an important and routinely performed procedure in spinal cord medicine. Individuals with spinal cord injury are at increased risk of hyper and hypothermia with exposure to extreme environmental temperatures. ${ }^{1}$ Also, elevation of body temperature often signals the onset of infection, a finding which results in clinical assessment, laboratory investigation and treatment intervention with treatment efficacy monitored using body temperature measurements.

Recently, the use of infrared (IR) tympanic membrane (TM) thermometers to measure body temperature has become popular. Many hospitals, including spinal cord units, use IR tympanic thermometers because they are quick and easy to use. They are used like an otoscope to seal the auricular canal, and an IR sensor gathers emitted IR energy for a preset amount of time 'primarily from the tympanic membrane'. ${ }^{2}$ This energy is then converted by a microprocessor into core, rectal and oral equivalents. They work on the assumption that the tympanic membrane temperature correlates closely with the core body temperature. This assumption has been validated in patients without spinal cord injury. Shinozaki et al $^{2}$ looked at a group of ICU patients using an IR tympanic thermometer (First Temp ${ }^{\circledR}$, Sherwood Intelligent Medical Systems, Carlsbad, California, USA) versus a pulmonary artery thermistor. They found that the IR thermometer

Correspondence: Dr Ansel Chu, Glenrose Rehabilitation Hospital, 10230-111 Avenue, Edmonton, Alberta T5G OB7, Canada tracked the core body temperature closely with a correlation coefficient of 0.98 over a temperature range of $34.0-39.5^{\circ} \mathrm{C}$. There was also excellent reproducibility between right and left TM temperatures. However, Jakobsson et $\mathrm{al}^{3}$ found that, even though there was excellent reliability for four different IR thermometers ( $r=0.96$ for First Temp), there was a systematic error in the conversion factor used to calculate temperature from IR energy. Two of the IR tympanic membrane thermometers gave consistently low and two consistently high readings compared to pulmonary artery temperature (First Temp over estimated core temperature by $0.5 \pm 0.2^{\circ} \mathrm{C}$ ). Heidenrich et $a l^{4}$ and Nierman et $a l^{5}$ also found similar results with the First Temp thermometer.

However, the disruption of autonomic control of temperature regulation in individuals with a high level spinal cord injury may result in differences in thermoregulatory function and temperature of the head compared to the rest of the body. ${ }^{6-8}$ Under normal circumstances, the hypothalamus functions as the control centre or 'thermostat' of the body. Afferent messages regarding body temperature reach the hypothalamus via the blood and from thermoreceptive nerve endings via the spinal cord. Messages from the hypothalamus to increase body temperature descend the brainstem and spinal cord to stimulate the sympathetic and somatic nerves resulting in shivering, cutaneous vasoconstriction and increased use of fat as an energy source. Conversely, messages from the hypothalamus to decrease body temperature descend the brainstem and spinal cord to stimulate peripheral vasodilatation and sweating. Spinal cord injury interrupts the flow of afferent input to the hypothalamus regarding skin 
temperature and efferent messages from the hypothalamus for shivering, sweating and vasoconstriction/ dilation below the level of the lesion. Thus, thermoregulation for individuals with high level spinal cord injuries is difficult and they tend to assume their environmental temperature and are thus termed 'poikilothermic'.

The part of the body above the level of the spinal cord injury (including head and tympanic membrane) remains responsive to the cholinergically mediated vasomotor and sudomotor adjustment commands of the hypothalamus whereas the portion of the body distal to the spinal cord injury level is unresponsive. For example, in a state of autonomic hyperreflexia, the head and neck of a tetraplegic person may be flushed and sweating whereas the trunk and lower extremities are pale and dry. This 'autonomic dissociation' was graphically observed in a previous study by one of the co-authors. ${ }^{6}$ Such a phenomenon may affect the validity of the concept that temperature measurements taken from the tympanic membrane accurately reflect core body temperature in individuals with a high spinal cord injury.

The research to date has, for the most part, demonstrated the reliability and validity of TM thermometers in measuring core body temperature in spinal intact subjects. Therefore the purpose of this study was to determine (1) the intrarater and intrasession reliability of an IR TM thermometer (First Temp-Genius) as well as rectal and oral temperature measures, and (2) the validity of IR tympanic and oral temperature measures in reflecting core body (rectal) temperature changes in individuals with high spinal cord injury.

\section{Patients and methods}

\section{Subjects}

A convenience sample of 11 subjects were recruited from the inpatient and outpatient clinics of the Glenrose Rehabilitation Hospital. All subjects had high spinal cord injuries ( $44 n=1, \mathrm{C} 6 n=6, \mathrm{C} 7 n=2$, T2 $n=1$, T4 $n=1)$, ASIA impairment scale classification of A (motor and sensory complete, $n=9$ ) or $\mathrm{B}$ (sensory incomplete, motor complete, $n=2$ ), and had signed an informed consent form.

\section{Methodology}

Temperature measurements were recorded using a First Temp-Genius IR tympanic thermometer (Intelligent Medical Systems, Carlsbad, California) set on rectal mode and a T-500 oral/rectal electronic thermometer. Calibration of the IR thermometer was done every 2 weeks during the course of the study as per the manufacturer's specifications by the hospital clinical engineering department. Calibration of the electronic thermometers was done using a water bath with comparison to a glass-mercury thermometer.

All temperature measurements were performed by the same tester. Measurements were taken for rectal, right TM, left TM and oral sites at $0,5,10$ and $15 \mathrm{~min}$ on two separate sessions (different days). The rectal probe was inserted intrarectally approximately $5 \mathrm{~cm}$ in depth. The oral probe was placed sublingually. The tympanic thermometer was seated firmly in the auricular canal, angled forward and a tug was applied to the helix of the ear to straighten out the canal.

Pearson's correlation coefficient was used to calculate intrasession reliability for rectal, right TM, left TM, oral and right versus left TM measures. A two way analysis of variance (type of thermometer $\times$ time of testing) for repeated measures (time of testing at 0 , $5,10,15 \mathrm{~min}$ ) was then used as well as descriptive statistic to generate mean and standard deviation values. Validity was calculated by comparing rectal to right TM, rectal to left TM and rectal to oral for both sessions 1 and 2 at the four different times using Pearson's correlation coefficient. Mean $r$ values were obtained by calculating the square root of the mean of the squared $r$ values.

\section{Results}

\section{Reliability}

Intrasession intratester reliability of the rectal, IR tympanic and oral thermometers are depicted in Table 1.

Repeated measures analysis of variance showed no significant differences for the main effects of time of testing $(0,5,10,15 \mathrm{~min})$ or type of thermometer (rectal, TM-rectal mode, oral) although the oral temperatures tended to be lower than the rectal and tympanic measures (Table 2).

\section{Validity}

The correlation between rectal temperature measures (gold standard) and those obtained from the tympanic and oral sites are summarized in Table 3 .

\section{Discussion}

This study was designed to evaluate the reliability and validity of TM temperature measurements in individuals with a high spinal cord injury. We found rectal temperatures to be the most reliable $(r=0.98)$ followed by oral $(r=0.88)$ which was slightly better than TM measurements $(r=0.86)$. However, looking at an internal check of reliability between right and left TM, there was only moderate correlation between the right and left sides. This may be because the tester was right-handed and measures from the left ear were more awkward and thus may have had more variability compared to the right (angle of insertion and amount of ear tug may affect the TM temperature readings). This suggests that tester technique may be a source of error and thus should be standardized, practiced and intratester reliability established $a$ priori. The presence of cerumen has been reported not to affect TM temperature readings. ${ }^{9}$ The $r$ value for the tympanic measures in this study is somewhat lower than those cited in other studies. ${ }^{2,3,5}$ This may be partly due to the narrower 
Table 1 Pearson's correlation coefficient at various sites

\begin{tabular}{llllll}
\hline $\begin{array}{l}\text { Session 1 } \\
\text { (sites) }\end{array}$ & $r^{a}$ & $\begin{array}{l}\text { Session 2 } \\
\text { (sites) }\end{array}$ & $r^{a}$ & Combined 1 and 2 \\
\hline Rectal & 0.97 & Rectal & 0.98 & Rectal & $r^{a}$ \\
Right TM & 0.84 & Right TM & 0.89 & Right TM & 0.975 \\
Left TM & 0.83 & Left TM & 0.88 & Left TM & 0.87 \\
& & & 0.92 & Left + right TM & 0.85 \\
Oral & 0.83 & Oral & 0.85 & Left vs right TM & 0.88 \\
Left vs right TM & 0.57 & Left vs right TM & & 0.72 \\
\hline
\end{tabular}

$\mathrm{TM}=$ infrared tympanic temperature thermometer

${ }^{a}$ All $r$ values statistically significant $(P<0.05)$

Table 2 Mean temperature measures \pm standard deviations for each type of thermometer (sessions 1 and 2 )

\begin{tabular}{lcccc} 
& $0 \mathrm{~min}$ & $5 \mathrm{~min}$ & $10 \mathrm{~min}$ & $15 \mathrm{~min}$ \\
\hline Session 1 & & & & $36.68 \pm 0.39$ \\
Rectal & $36.72 \pm 0.44$ & $36.69 \pm 0.43$ & $36.69 \pm 0.42$ & $36.67 \pm 0.60$ \\
R TM & $36.47 \pm 0.63$ & $36.65 \pm 0.60$ & $36.71 \pm 0.59$ & $36.80 \pm 0.51$ \\
L TM & $36.83 \pm 0.43$ & $36.75 \pm 0.51$ & $36.72 \pm 0.46$ & $36.29 \pm 0.45$ \\
Oral & $36.34 \pm 0.41$ & $36.25 \pm 0.43$ & & $36.85 \pm 0.43$ \\
Session 2 & & & $36.83 \pm 0.45$ & $36.89 \pm 0.67$ \\
Rectal & $36.87 \pm 0.45$ & $36.84 \pm 0.44$ & $36.86 \pm 0.67$ & $36.82 \pm 0.55$ \\
R TM & $36.66 \pm 0.74$ & $36.81 \pm 0.66$ & $36.67 \pm 0.52$ & $36.39 \pm 0.50$ \\
L TM & $36.64 \pm 0.54$ & $36.65 \pm 0.56$ & $36.32 \pm 0.57$ & \\
Oral & $36.25 \pm 0.63$ & $36.38 \pm 0.54$ & & \\
\hline
\end{tabular}

$\mathrm{TM}=$ infrared tympanic thermometer.

All values statistically insignificant $(P>0.05)$

Table 3 Correlation coefficients of rectal to tympanic and oral thermometers

\begin{tabular}{|c|c|c|c|c|c|c|}
\hline Times & $\begin{array}{c}R T M \\
\text { session } \\
1\end{array}$ & $\begin{array}{c}L T M \\
\text { session } \\
1\end{array}$ & $\begin{array}{c}\text { Oral } \\
\text { session } \\
1\end{array}$ & $\begin{array}{c}R T M \\
\text { session } \\
2\end{array}$ & $\begin{array}{c}L T M \\
\text { session } \\
2\end{array}$ & $\begin{array}{c}\text { Oral } \\
\text { session } \\
2\end{array}$ \\
\hline $0 \mathrm{~min}$ & 0.77 & 0.15 & 0.74 & 0.63 & 0.84 & 0.83 \\
\hline $5 \mathrm{~min}$ & 0.66 & 0.29 & 0.89 & 0.73 & 0.73 & 0.90 \\
\hline $10 \mathrm{~min}$ & 0.90 & 0.44 & 0.95 & 0.79 & 0.85 & 0.89 \\
\hline $15 \mathrm{~min}$ & 0.80 & 0.41 & 0.85 & 0.80 & 0.72 & 0.95 \\
\hline $\begin{array}{l}\text { Combined } \\
\quad 0,5,10,15\end{array}$ & 0.79 & 0.34 & 0.86 & 0.74 & 0.79 & 0.89 \\
\hline $\begin{array}{l}\text { Combined } \\
\text { sessions } 1+2\end{array}$ & 0.77 & 0.61 & 0.88 & & & \\
\hline
\end{tabular}

range of temperatures in this group of subjects studied $\left(35.8-37.6^{\circ} \mathrm{C}\right)$ whereas the able bodied studies had a wider range $\left(34.0-39.5^{\circ} \mathrm{C}\right.$ rectal $\left.^{2}\right)$. It is statistically more difficult to obtain a high $r$ value within a narrower range of values.

The next question that we looked at was the validity of the tympanic measure compared to the rectal (gold standard) as well as oral compared to rectal. The oral measure appears to reflect more accurately changes in the rectal temperature with an $r$ of 0.88 compared to the right TM $r$ of 0.77 (Table 3). However, neither are as good as the validity reported by Shinozaki et al. ${ }^{2}$ Again, the narrow range of temperatures tested may be an explanation, but autonomic dissociation in the high spinal cord injured, which would affect both oral and tympanic measures when compared to rectal measures, may also be a factor. The correlation coefficient of the left TM temperatures compared to rectal temperatures was 0.34 overall for session 1 and 0.79 for session 2 . This poor correlation can also be seen in the right versus left TM $r$ value of 0.57 for session 1 and may reflect the awkwardness of taking a reading from the left TM using the right hand which may have improved with practice by session 2 . Assuming the right TM measurements accurately reflects the validity of the device, one can appreciate the increased variance of the TM measurements as compared to the oral measurements as the right TM standard deviation is $0.15^{\circ} \mathrm{C}$ 
greater than oral and $0.21^{\circ} \mathrm{C}$ greater than rectal. From a clinical perspective this small amount of increased variance is probably acceptable and would not affect clinical decision making. The time and cost saving of the IR TM device justifies its use. ${ }^{10}$

From previous studies ${ }^{3,4}$ it was shown that the First Temp ${ }^{\circledR}$ had a constant error of $+0.5^{\circ} \mathrm{C}$ compared to a reference (both studies used a pulmonary artery thermistor as the gold standard with the TM thermometer set on core temperature).. In this study we used a new generation TM thermometer (First Temp-Genius) which reportedly corrects this software calibration error (personal communication with Marlene Day, the technical representative for Intelligent Medical Systems). From Table 2 we see that there is no statistically significant difference between TM and rectal measures although there is a trend for TM readings being minimally lower than rectal (TM thermometer set on rectal mode). The calibration error seen previously with First Temp seems to have been corrected with the new generation TM thermometer.

One question that was not addressed in this study was what happens to the validity of tympanic and oral measures at higher temperatures (febrile state). The wider range of measures may strengthen the correlation. On the other hand, in a physical stress situation, such as a febrile illness, the consequences of autonomic dissociation may be more marked thus reducing the validity of the TM measurements. At higher temperatures a difference of $0.2^{\circ} \mathrm{C}$ may be more clinically significant to the diagnosis of a febrile illness. Further studies in this population of subjects during febrile states will be necessary to elucidate these points.

The reliability and validity of the infrared tympanic thermometer is slightly worse than oral but is well within acceptable limits in the clinical setting for patients with high spinal cord injuries. Within the afebrile state the tympanic measure accurately reflects the core body temperature as measured by the rectal thermometer.

\section{Acknowledgements}

Funding for this study was exclusively provided by a grant from the Glenrose Research Fund. Invaluable feedback and suggestions were provided by the physiatry staff at the Glenrose Rehabilitation Hospital.

\section{References}

1 Bloch RF. Autonomic dysfunction. In Bloch RF (ed). Management of Spinal Cord Injuries. Williams and Wilkins, Baltimore and London, 1986, pp 149-163.

2 Shinozaki T, Deane R, Perkins F. Infrared tympanic thermometer: evaluation of a new clinical thermometer. Crit Care Med 1988; 16: 148-150.

3 Jakobsson J, Nilsson A, Carlsson L. Core temp measured in the auricular canal: comparison between four different thermometers. Acta Anaes Scand 1992; 36: 819-824.

4 Heidenreich T, Giuffre M, Doorley J. Temperature and temperature measurement after induced hypothermia. Nursing Res 1992; 41: 296-300.

5 Nierman D. Core temperature measurement in the intensive care unit. Crit Care Med 1991; 16: 818-823.

6 Burnham R. Intentional induction of autonomic dysreflexia among quadriplegic athletes for performance enhancement: efficacy, safety and mechanism of action. Clin J Sports Med (in press).

7 Guttmann L, Silver J, Wyndham CH. Thermoregulation in spinal man. J Physiol 1958; 142: 406-419.

8 Normell CA. Distribution of impaired cutaneous vasomotor and sudomotor function on paraplegic man. Scand $J$ Lab Invest 1974; 33(S138): 25-41.

9 Phillips DB. The physiologic rationale for the ear as an ideal site for core temperature assessment. Presented at the Seventh Annual Spring Head and Neck Workshop, March 85, Snowbird, Utah.

10 Alexander J. Cost effectiveness of tympanic thermometry in the pediatric office setting. Clin Pediatr (Philadelphia) 1991; 30: 57-59. 